\title{
The Deconditioning Effect of the COVID-19 Pandemic on Unaffected Healthy Children
}

\author{
Jeffrey D. Dayton ${ }^{1,2} \cdot$ Kelley Ford $^{1,2} \cdot$ Sheila J. Carroll ${ }^{1,2} \cdot$ Patrick A. Flynn $^{1,2} \cdot$ Soultana Kourtidou $^{1,2} \cdot$ Ralf J. Holzer $^{1,2}$
}

Received: 15 October 2020 / Accepted: 20 November 2020 / Published online: 4 January 2021

(c) Springer Science+Business Media, LLC, part of Springer Nature 2021

\begin{abstract}
The COVID-19 pandemic has had devastating direct consequences on the health of affected patients. It has also had a significant impact on the ability of unaffected children to be physically active. We evaluated the effect of deconditioning from social distancing and school shutdowns implemented during the COVID-19 pandemic on the cardiovascular fitness of healthy unaffected children. This is a single-center, retrospective case-control study performed in an urban tertiary referral center. A cohort of 10 healthy children that underwent cardiopulmonary exercise testing after COVID-19 hospital restrictions were lifted was compared to a matched cohort before COVID-19-related shutdowns on school and after-school activities. Comparisons of oxygen uptake $\left(\mathrm{VO}_{2}\right)$ max and $\mathrm{VO}_{2}$ at anaerobic threshold between the pre- and post-COVID-19 cohorts were done. The $\mathrm{VO}_{2}$ max in the post-COVID cohort was significantly lower than in the pre-COVID cohort (39.1 vs. 44.7, $p=0.031$ ). Only one out of ten patients had a higher $\mathrm{VO}_{2} \max$ when compared to their matched pre-COVID control and was also the only patient with a documented history of participation in varsity-type athletics. The percentile of predicted $\mathrm{VO}_{2}$ was significantly lower in the post-COVID cohort $(95 \%$ vs. $105 \%, p=0.042)$. This study for the first time documented a significant measurable decline in physical fitness of healthy children as a result of the COVID-19 pandemic and its associated restrictions. Measures need to be identified that encourage and facilitate regular exercise in children in a way that are not solely dependent on school and organized after-school activities.
\end{abstract}

Keywords Functional capacity $\cdot \mathrm{VO}_{2} \cdot \mathrm{COVID}-19$

$\begin{array}{ll}\text { Abbreviations } \\ \text { CPET } & \text { Cardiopulmonary exercise test } \\ \mathrm{VO}_{2} \text { max } & \text { Maximal oxygen uptake } \\ \mathrm{BMI} & \text { Body mass index } \\ \text { PCR } & \text { Polymerase chain reaction } \\ \text { RER } & \text { Respiratory exchange ratio } \\ \text { EKG } & \text { Electrocardiogram } \\ \text { VE } & \text { Ventilation } \\ \mathrm{VCO}_{2} & \text { Carbon dioxide production } \\ \mathrm{PETCO}_{2} & \text { Partial pressure of end-tidal carbon dioxide }\end{array}$

Electronic supplementary material The online version of this article (https://doi.org/10.1007/s00246-020-02513-w) contains supplementary material, which is available to authorized users.

Ralf J. Holzer

rjh3001@med.cornell.edu

1 Division of Pediatric Cardiology, Weill Cornell Medicine, 525 East 68th Street, Room F-677, New York, NY 10065, USA

2 New York-Presbyterian Komansky Children's Hospital, New York, NY, USA

\section{Introduction}

The COVID-19 pandemic has had devastating direct consequences on the health of affected patients. Furthermore, it has also had a multitude of secondary consequences on the economy and education. Children were required to attend school remotely for several months and were therefore unable to engage in their regular school, social, and after-school activities including physical education and sports programs. While the impacts on education have been tremendous, what has not been evaluated are the equally important potential implications on the health and cardiovascular fitness of unaffected children and young adults as a result of the COVID-19-related shutdown which limited opportunities to partake in regular physical activity at school, recreational sports, and competitive athletics. Deleterious effects due to physical inactivity can include loss of muscle mass within days as well as impairment in aerobic capacity due to home confinement [1]. This may be of particular importance in large urban areas that were heavily affected early in the pandemic on which stricter social distancing and other control 
measures were implemented to contain the spread of the virus. As an example, in New York City, school closures were implemented on March 16, 2020 while strict social distancing measures currently remain in-place. Commercial gyms were allowed to re-open five months later on August 24, 2020 and public schools began to re-open in New York City on September 21, 2020 at the beginning of the new school year. However, this reopening has included a hybrid model of remote and in-person instruction.

In these large urban areas, the pandemic may have resulted in a significant reduction in the cardiovascular fitness of children and young adults which could have significant long-term implications. Therefore, to better understand the impact of the COVID-19 shutdown of school and recreational activities on the cardiovascular fitness of children and young adults, this study evaluates the exercise performance of children and young adults 3-6 months into the pandemic and its associated shutdowns, and compares it to the exercise performance of a matched cohort before these restrictions were implemented.

\section{Methods}

A retrospective study was performed at a single New York City urban tertiary referral center located in the middle of Manhattan, comparing exercise capacity in ten healthy children and young adults who underwent cardiopulmonary exercise testing (CPET) on or after the reopening of the exercise laboratory on June 15, 2020 but before the official date for re-starting school activities in New York City on September 21,2020, to ten matched controls who underwent exercise testing prior to the shutdown of the exercise laboratory on March 19, 2020. This study was reviewed by our Institutional Review Board and deemed exempt.

\section{Primary Objectives}

- To compare the functional capacity derived by cardiopulmonary exercise testing pre-COVID to a matched cohort several months into the COVID pandemic

\section{Inclusion Criteria}

- Any patient that underwent cardiopulmonary exercise testing after June 15, 2020 (when the cardiology division resumed CPET)

- Matched controls were included if the following criteria were fulfilled (criteria were chosen based on previously published data on parameters that affect $\mathrm{VO}_{2} \max$ [2-7])
- Cardiopulmonary exercise testing within the prior 2 years

- Gender

- Age \pm 1 year

- Match by race (Hispanic, White, Black, Asian, Other)

- Body mass index $\pm 10 \%$

- Height and weight: $\pm 10 \%$

- If a perfect match could not be identified, criteria were expanded to allow the following:

- Age \pm 2 years

- Match by race:

- Hispanic and whites were considered one group

- Patients that declined to provide ethnicity were considered an appropriate match for any race

- Height and weight:

- One criterion of the two was allowed to extent to $\pm 15 \%$, provided the BMI was within a \pm $10 \%$ range

- Matching procedure:

- The first matched control was identified by sequentially screening all CPET from newest to oldest

- A control that was matched to one patient could not be matched again to another, even if considered a perfect match

\section{Exclusion Criteria}

- Any significant comorbidity that is expected to affect cardiopulmonary exercise performance

- Patients with a history of documented COVID-19 infection (either by antibody serology or PCR) at the time of stress testing

- Age above 21 years

- Patients undergoing exercise testing at or after official reopening of schools in New York City (September 21, 2020)

- Patients that do not have a match with adequate matching criteria

- Submaximal stress test with respiratory exchange ratio (RER) $<1.1$

- Patients with significant EKG changes during CPET 
- Abnormal baseline echocardiography documenting either a structural cardiac anomaly, or any abnormality of systolic or diastolic function

\section{Outcome Parameters and Predictors}

This is a retrospective case-control study evaluating for a possible decline in exercise performance of healthy pediatric patients following 3-5 months of COVID-19 restrictions. The following data were collected:

- Basic demographic data age, gender, height, weight, BMI, ethnicity

- Clinical data comorbidities, participation in varsity sports (four or more times a week, participating in competitions), baseline echocardiography

- Stress test data type of test, $\mathrm{VO}_{2} \max$ (and percent of predicted $\left.\mathrm{VO}_{2} \max \right), \mathrm{VO}_{2}$ at the anaerobic threshold, maximum heart rate (and \% of predicted maximum heart rate), maximum respiratory exchange ratio during exercise, heart rate reserve, $\mathrm{VE} / \mathrm{VCO}_{2}, \mathrm{PETCO}_{2}$

\section{Stress Test Protocols}

All patients underwent cardiopulmonary stress testing in a single tertiary referral center supervised by the same cardiologists. In the post-COVID cohort, SARS-CoV-2 PCR testing was required within 5 days prior to testing and exercise testing included the use of appropriate personal protective equipment for the staff performing the study. Patients underwent either a Vancouver protocol [8] or a Bruce protocol. The Vancouver protocol allowed smaller incremental changes in the workload, but has the disadvantage of making blood pressure recordings at faster treadmill speeds more difficult when compared to the Bruce protocol. Preand post-exercise spirometry were obtained only in selected patients. Therefore, parameters that depend on a more accurate assessment of spirometry data rather than estimates (such as the maximal voluntary ventilation and breathing reserve) are not provided for all patients.

For all patients in this study, stress tests were continued until patients reached either their maximum predicted heart rate with an RER $>1.1$, or when a deteriorating running form required discontinuation of the protocol. No patient in this study required early termination due to concerning clinical symptoms, EKG changes, or blood pressure abnormalities.

\section{Statistics}

Pre- and post-COVID cohorts were compared with regard to age, height, weight, BMI, gender, ethnic origin, and type of test performed to identify whether the cohorts are similar with respect to these variables using a paired $t$ test for discrete variables and chi-square test for categorical variables. The cohorts were also compared for important stress test parameters to identify whether there were any significant differences between these two groups relating to the level of exertion (maximum heart rate, max RER during exercise) using a two-sided paired $t$ test.

To evaluate whether there was a decline in the postCOVID cohort for exercise performance, a one-sided paired $t$ test was used to compare $\mathrm{VO}_{2} \max$ and $\mathrm{VO}_{2}$ at anaerobic threshold between pre- and post-COVID cohorts.

\section{Results}

\section{Patient Cohorts}

The exercise laboratory for the division of pediatric cardiology at Weill Cornell Medicine reopened to perform CPETs on June 15, 2020 after institutional limitations on non-urgent procedures were lifted following the COVID-19 surge. Schools in New York began reopening on September 21, 2020, while commercial gyms were allowed to open a month earlier on August 24, 2020. During the time period between resumption of CPET testing at our institution and the reopening of school (June 15, 2020 through September 21, 2020), 22 cardiopulmonary stress tests were performed. Seven patients had some exclusion criteria to participating in this study: four patients had congenital heart disease, one patient had hypertrophic obstructive cardiomyopathy (HOCM), one patient had a history of COVID-19 infection, and one patient had a submaximal effort during exercise testing. An additional five patients did not have a patient identified that fulfilled the matching criteria. Out of the remaining ten patients, six fulfilled all matching criteria, while four required the extended criteria.

\section{Clinical Parameters that Impact $\mathrm{VO}_{2}$ and $\mathrm{VO}_{2}$ Max}

Basic demographic and clinical data are listed in Table 1 (and supplemental Tables 1 and 2). There were no significant differences relating to gender, race, weight, height, BMI, and procedural indication. The mean age in the post-COVID cohort was slightly higher than in the pre-COVID cohort ( 15.2 vs. 14.5 years, $p=0.025$ ). Out of 20 patients, only one had a clear documentation of exercising more than four times a week at a competitive level. The most common indication for CPET was chest pain (7/20), followed by electrophysiological indications such as Wolff-Parkinson-White (WPW), borderline prolonged QT interval, palpitations, supraventricular tachycardia (5/20), and shortness of breath (5/20). Additional indications included (pre)syncope (3/20).

Four out of the ten patients in the pre-COVID cohort underwent CPET using the Vancouver protocol, while all 
Table 1 Basic demographic and clinical data

\begin{tabular}{llll}
\hline & Pre-COVID & Post-COVID & $p$ value \\
\hline Gender & & & \\
$\quad$ Male & 4 & 4 & 1.0 \\
$\quad$ Female & 6 & 6 & \\
Race & & 6 & 0.23 \\
$\quad$ White & 9 & 2 & \\
Hispanic & & 2 & 0.025 \\
Unspecified & 0 & $15.2( \pm 3.2)$ & 0.78 \\
Age (years) & $14.5( \pm 3.2)$ & $66( \pm 6.4)$ & 0.44 \\
Height (inches) & $66( \pm 5.2)$ & $131( \pm 34.1)$ & 0.11 \\
Weight (lbs) & $128( \pm 30.5)$ & $20.9( \pm 2.9)$ & \\
BMI (kg/m $\left.{ }^{2}\right)$ & $20.2( \pm 2.6)$ & & 0.83 \\
Indication & & 3 & \\
Chest pain & 4 & 3 & \\
SOB & 2 & 3 & \\
(Pre) syncope & 2 & 2 &
\end{tabular}

EP Electrophysiology

patients in the post-COVID cohort were tested using the Bruce protocol. There were no significant differences in important cardiovascular and pulmonary data such as maximum heart rate, percentage of predicted maximum heart rate, RER at peak exercise, heart rate reserve, $\mathrm{VE} / \mathrm{VCO}_{2}$, and $\mathrm{PETCO}_{2}$ between the pre-COVID and the post-COVID cohorts (Table 2, Supplemental Table 3a, b).

\section{Assessment for a Decline in Exercise Performance}

Results of exercise performance are listed in Table 3 (and Supplemental Table 4). The $\mathrm{VO}_{2}$ max in the post-COVID cohort was significantly lower than in the pre-COVID cohort (39.1 vs. 44.7, $p=0.031$ ). In fact, only one patient out of ten had a higher value in the $\mathrm{VO}_{2}$ max when compared to matched pre-COVID controls. This was the only patient with a documented history of participation in varsity-level

Table 2 Basic CPET data

\begin{tabular}{llll}
\hline & Pre-COVID & Post-COVID & $p$ value \\
\hline CPET protocol & & & \\
$\quad$ Bruce & 6 & 10 & 0.025 \\
$\quad$ Vancouver & 4 & 0 & \\
Maximum HR $(b p m)$ & $195( \pm 8.4)$ & $195( \pm 9.8)$ & 0.95 \\
\% of predicted max HR & $94.6( \pm 3.6)$ & $94.7( \pm 3.3)$ & 0.95 \\
RER at $\mathrm{VO}_{2}$ max & $1.28( \pm 0.08)$ & $1.31( \pm 0.1)$ & 0.41 \\
Heart rate reserve $(\%)$ & $4.8( \pm 3.4)$ & $5( \pm 3.7)$ & 0.91 \\
VE/ $\mathrm{VCO}_{2}$ & $31.9( \pm 3.8)$ & $31.7( \pm 3.7)$ & 0.89 \\
PETCO $_{2}$ & $37( \pm 1.7)$ & $38.1( \pm 1.5)$ & 0.37 \\
\hline
\end{tabular}

Table 3 CPET exercise performance data

\begin{tabular}{lcll}
\hline & Pre-COVID & Post-COVID & $p$ value \\
\hline $\mathrm{VO}_{2}$ max & $44.7( \pm 7.7)$ & $39.1( \pm 5.2)$ & 0.031 \\
$\mathrm{VO}_{2}$ max \% predicted & $105( \pm 14.2)$ & $94.6( \pm 10.4)$ & 0.042 \\
Anaerobic threshold & $24.6( \pm 5.5)$ & $21.5( \pm 4.1)$ & 0.082 \\
\hline
\end{tabular}

activities, exercising more than four times a week at a competitive level (and continuing to do so during the COVIDrelated shutdowns). The percentile of predicted $\mathrm{VO}_{2}$ was significantly lower in the post-COVID cohort (95\% vs. $105 \%$, $p=0.042$ ). There was a trend for the anaerobic threshold to be lower in the post-COVID cohort, even though it did not reach statistical significance (21.5 vs. $24.6, p=0.082)$.

\section{Discussion}

This study has documented a significant decrease in functional capacity and anaerobic threshold when comparing a post-COVID cohort to matched pre-COVID controls. In fact, there was only one out of ten paired patients in whom the $\mathrm{VO}_{2}$ in the post-COVID patient was higher than their preCOVID control. Also, this was the only patient out of twenty with a clearly documented history of competitive varsitytype sports activity (dancing) that was also continued during the COVID-19-related shutdowns. There were no significant cardiovascular or pulmonary differences between the two groups that could account for the differences observed.

This study has for the first time documented a measurable effect of the COVID-19 pandemic on the exercise capacity of healthy children, as reflected in the decline of $\mathrm{VO}_{2}$ max. SARS-CoV-2 has had a devastating effect on patients directly affected by the virus but there are also indirect effects on society as a whole due to social distancing measures and school-related shutdowns. Children and their need for exercise have not been spared by the effects of these measures. While adults are often accustomed to independent exercise, the majority of children receive their main exposure to exercise through school and recreational activities, as well as important social activities such as summer camps during school breaks. A study by Drake et al. showed that participation in team sports was inversely related to being overweight/obese in high school students [9]. All of these activities have been extremely restricted during the COVID19 pandemic. In a Canadian study, it was found that only $4.8 \%$ of children were meeting the movement behaviors guidelines during the COVID-19 pandemic [10]. Replacing these activities with safe alternatives in the setting of the COVID-19 pandemic is essential to promoting healthy habits while reducing the risk for obesity from a sedentary lifestyle. 
At a young age, foundations are often laid for developing healthy long-term behaviors. An expert panel convened by the American Academy of Pediatrics in 2011 reported that there is "reasonably good evidence" that physical activity patterns formed in childhood are continued in adulthood. This panel also concluded that there is "strong evidence" for favorable clinical effects from physical activity in childhood and adolescence with regard to blood pressure, body fat, body mass index, and cholesterol levels [11]. Therefore, there are favorable effects from exercise in childhood that can extend into adulthood but discontinuation of these activities during the pandemic has the potential to disrupt these habits that children would otherwise continue as adults. Eliminating the ability to exercise could potentially lead not only to the short-term decline of $\mathrm{VO}_{2}$ max that has been documented in this study, but also a loss of the understanding of the importance of physical activity and exercise. With the ongoing COVID-19 pandemic and its associated restrictions that will likely remain in-place for the near future, it is even more important to identify ways to allow children to safely participate in physical activities. While remote learning can replace some of children's educational needs, online classes will not be able to replace the ability to exercise. Novel activities such as the Peleton bike provide socially interactive ways to potentially engage children in cardiovascular exercise from home, but affordability and space requirements are often prohibitive factors for many families. While these options can improve access to exercise in a safe manner during a pandemic, there is the potential for these methods to contribute to the social inequities exposed by the COVID-19 pandemic if access is limited to those of higher socioeconomic status [12] including children [13]. Participation in vigorous family activities may be one approach to increase physical activity while minimizing additional potential infectious exposure risk [14]. In the long-term, strategies need to be identified that encourage regular exercise for all children in a way that is not solely dependent on school and organized after-school activities.

\section{Limitations}

This study has several limitations. Most importantly is the small sample size, which was limited to exercise tests performed prior to reopening of schools following COVID19-related shutdowns. Furthermore, these comparisons of exercise performance were not obtained on the same patient pre- and post-COVID but instead matched controls and, therefore, the observed differences could have occurred by chance. Moreover, two patients had unspecified ethnicity which could potentially have impacted the results given race-related differences in $\mathrm{VO}_{2}$ max. [2, 3, 6] In addition, several patients in the pre-COVID cohort had a more graded exercise protocol (Vancouver) [8] in comparison to the post-COVID cohort. The Vancouver protocol had been evaluated for several months at our institution but the higher treadmill speed was less suitable for manual blood pressure measurement during the test, because of which we have since reverted back to the Bruce protocol for the majority of patients at our center.

\section{Summary}

For the first time, this study has documented a significant measurable decline in physical fitness of healthy children due to the COVID-19 pandemic and its associated restrictions. Measures need to be identified that encourage and facilitate regular exercise in children in ways that are not solely dependent on school and organized after-school activities.

Data Availability Available upon reasonable request.

\section{Compliance with Ethical Standards}

Conflict of Interest The authors declared that they have no conflict of interest to disclose.

Ethical Approval This study was reviewed by our institution's Institutional Review Board and deemed exempt.

\section{References}

1. Narici M, De Vito G, Franchi M et al (2020) Impact of sedentarism due to the COVID-19 home confinement on neuromuscular, cardiovascular and metabolic health: physiological and pathophysiological implications and recommendations for physical and nutritional countermeasures. Eur J Sport Sci. https://doi. org/10.1080/17461391.2020.1761076

2. Andreacci JL, Robertson RJ, Dube JJ, Aaron DJ, Balasekaran G, Arslanian SA (2004) Comparison of maximal oxygen consumption between black and white prepubertal and pubertal children. Pediatr Res 56(5):706-713

3. Andreacci JL, Robertson RJ, Dube JJ, Aaron DJ, Dixon CB, Arslanian SA (2005) Comparison of maximal oxygen consumption between obese black and white adolescents. Pediatr Res 58(3):478-482

4. Carrick-Ranson G, Hastings JL, Bhella PS et al (2013) The effect of age-related differences in body size and composition on cardiovascular determinants of $\mathrm{VO}_{2}$ max. J Gerontol A 68(5):608-616

5. Mondal H, Mishra SP (2017) Effect of BMI, body fat percentage and fat free mass on maximal oxygen consumption in healthy young adults. J Clin Diagn Res 11(6):CC17-CC20

6. Nightingale CM, Donin AS, Kerry SR et al (2016) Cross-sectional study of ethnic differences in physical fitness among children of South Asian, black African-Caribbean and white European origin: the Child Heart and Health Study in England (CHASE). BMJ Open 6(6):e011131

7. Wang C, Haskell WL, Farrell SW et al (2010) Cardiorespiratory fitness levels among US adults 20-49 years of age: findings from 
the 1999-2004 National Health and Nutrition Examination Survey. Am J Epidemiol 171(4):426-435

8. Duff DK, De Souza AM, Human DG, Potts JE, Harris KC (2017) A novel treadmill protocol for exercise testing in children: the British Columbia Children's Hospital protocol. BMJ Open Sport Exerc Med 3(1):e000197

9. Drake KM, Beach ML, Longacre MR et al (2012) Influence of sports, physical education, and active commuting to school on adolescent weight status. Pediatrics 130(2):e296-304

10. Moore SA, Faulkner G, Rhodes RE et al (2020) Impact of the COVID-19 virus outbreak on movement and play behaviours of Canadian children and youth: a national survey. Int J Behav Nutr Phys Act 17(1):85

11. Expert Panel on Integrated Guidelines for Cardiovascular Health and Risk Reduction in Children and Adolescents, National Heart Lung and Blood Institute (2011) Expert panel on integrated guidelines for cardiovascular health and risk reduction in children and adolescents: summary report. Pediatrics 128(5):213-256
12. Webb Hooper M, Napoles AM, Perez-Stable EJ (2020) COVID-19 and racial/ethnic disparities. JAMA 323(24):2466-2467

13. Goyal MK, Simpson JN, Boyle MD et al (2020) Racial and/or ethnic and socioeconomic disparities of SARS-CoV-2 infection among children. Pediatrics 146(4):e2020009951

14. Keeping Children Active during the Coronavirus Pandemic [Internet] (2020) Exercise is Medicine. American College of Sports Medicine, Indianapolis. https://www.exerciseismedicine.org/asset s/page_documents/EIM_Rx\%20for\%20Health_\%20Keeping\%20 Children\%20Active\%20During\%20Coronavirus\%20Pandemic .pdf. Accessed 2 Oct 2020

Publisher's Note Springer Nature remains neutral with regard to jurisdictional claims in published maps and institutional affiliations. 